Unfallchirurg 2013 $\cdot 116: 870-871$

DOI 10.1007/s00113-013-2442-x

Online publiziert: 8. Oktober 2013

(c) Springer-Verlag Berlin Heidelberg 2013
P.C. Strohm ${ }^{1} \cdot$ H. Siebert ${ }^{2}$

${ }^{1}$ Department Chirurgie, Klinik für Orthopädie und Unfallchirurgie, Albert-Ludwigs-Universität Freiburg

${ }^{2}$ Geschäftsstelle Deutsche Gesellschaft für Orthopädie und Unfallchirurgie e.V., Berlin

\title{
Wie können wir die Patientensicherheit verbessern?
}

Die Zahl der auf vermeidbare Fehler zurück zu führenden Todesfälle in deutschen Krankenhäusern wurde nach einer Auswertung des deutschen Sachverständigenrates zur Begutachtung der Entwicklung des Gesundheitswesen 2007 auf der Basis einer Auswertung von 184 Studien „nur“ mit 0,1\% (von 1,7 Mio.) angeben, immerhin bedeutet dies 17.000 Todesfälle/ Jahr. In der Gesundheitsberichterstattung des Bundes 2011 werden auf 100.000 Einwohner 23,2 vermeidbare Todesfälle im Rahmen der medizinischen Versorgung angegeben. Als Folge von nosokomialen Infektionen sterben jährlich 10.000 15.000 Patienten in deutschen Kliniken. Diese Tatsachen sind mit ein Grund dafür, dass das Thema Patientensicherheit in der Gesundheitsversorgung international und auch in Deutschland in den letzten Jahren zunehmend an Bedeutung gewonnen hat. Um diesem Thema angemessen gerecht zu werden wurde 2005 das Aktionsbündnis Patientensicherheit gegründet (http://www.aps-ev.de/home/), welches seitdem Projekte zur Förderung der Patientensicherheit initiiert, unterstützt und durchführt. Es steht v. a. dafür ein, dass Fehler nicht tabuisiert, skandalisiert und nur „personalisiert" werden, sondern dass alle Beteiligten im Rahmen einer neuen Sicherheitskultur unerwünschte Ereignisse zum Anlass nehmen, um die Ursachen zu ergründen und präventive Maß-

H. Siebert ist Generalsekretär DGU/DGOU, stellvertretender Vorsitzender Aktionsbündnis Patientensicherheit - APS e.V. nahmen einzuführen (strategisches Risikomanagement).

Trotz des zunehmenden Interesses an einer Verbesserung der Patientensicherheit wird im medizinischen Bereich immer noch häufig die tradierte skeptische bis ablehnende Haltung angetroffen, z. B. mit der Einstellung „ich bin gut genug, Fehler passieren mir nicht, ich muss deshalb auch keine Vorsorge treffen und z. B. Kurse machen“. In vielen Abteilungen und Köpfen wird so noch eine sog. „culture of blame“ gepflegt, d. h. nach einem Fehler oder unerwünschten Ereignis wird der Schuldige gesucht und ,bestraft“. Die Nachhaltigkeit und der Lerneffekt eines solchen Vorgehens sind sehr gering. Werden nach längerer Zeit Beteiligte $\mathrm{zu}$ einem solchen Ereignis befragt, wissen die meisten noch, wer Schuld war, aber was zu dem Fehler geführt hat, ist in der Regel vergessen. Auch der offene Umgang und Austausch über unerwünschte Ereignisse wird - möglicherweise aus Furcht vor Sanktionen oder weil es der jeweiligen Person/Institution unangenehm ist - selten gepflegt, obwohl Studien zeigen, dass in Kliniken in denen ein Fehlerwarnsystem („critical incident reporting systems“, CIRS) im Rahmen des klinischen Risikomanagements besteht und genutzt wird, die Rate an unerwünschten Ereignissen abnimmt. Noch ist die Zahl der Einrichtungen, die dieses Instrument nutzen, gering, wiewohl die Zahl der Meldungen und der spezifischen und nationalen Datenbanken stetig zunimmt.
Dabei ist die Idee, aus fremden Fehlern zu lernen und damit eigene Fehler zu vermindern, doch charmant und elementarer Bestandteil des Lernens und Lehrens.

Diese Erkenntnis verwenden Unternehmen in Hochsicherheitsbereichen wie z. B. der Luftfahrt, welche sich schon lange mit der Analyse und Vermeidung von Fehlern beschäftigen. Laut Focus wurden 2012 rund 2,8 Mrd. Passagiere transportiert und knapp 40 Mio. Flüge durchgeführt, die Zahl der Todesopfer lag bei 496, die Zahl der Flugzeugtotalverluste bei 44 . Spezifische Instrumente wie Simulationstrainings, Checklisten usw. sind in der Luftfahrt - nachweislich mit Erfolg - schon lange Standard und finden langsam auch den Weg in unsere medizinische Arbeitswelt. Dies gelingt aber nur dann, wenn im Rahmen des strategischen Risikomanagements die Organisation Krankenhaus und damit auch die Geschäftsführung ihre Verantwortung erkennt und Maßnahmen zur Umsetzung und v. a. Aufrechterhaltung eines hohen Sicherheitsstandards ergreift. Wie ausgeprägt dieses Defizit in deutschen Krankenhäusern noch ist, zeigen die Ergebnisse einer Umfrage des APS 2011 zur Umsetzung des klinischen Risikomanagements (http://www.aps-ev.de/Publikationen).

Im neuen Patientenrechtegesetz wurde auf diese Mitverantwortung der Klinikgeschäftsführungen im Rahmen der Haftung besonders hingewiesen. Dies zeigt, dass auch in der Rechtsprechung so langsam neben einem „personifizierten Feh- 
lerverständnis“ die „systemische Verantwortung " berücksichtigt wird.

Der komplexe Tatbestand Fehlervermeidung wird in den folgenden Artikeln aus den verschiedenen Perspektiven dargestellt und Möglichkeiten zu deren Verbesserung in der Praxis aufgezeigt.

Wir hoffen, Sie anzuregen, sich mit dem Thema auseinander zu setzen, Ihnen bekannte Möglichkeiten zu nutzen, aber auch über weitere Möglichkeiten nachzudenken, um die Patientensicherheit künftig wirkungsvoll mit zu gestalten.
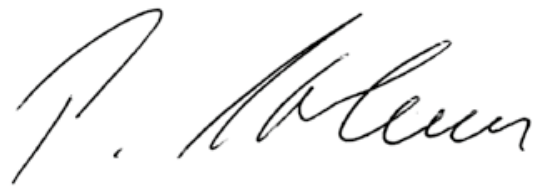

P.C. Strohm

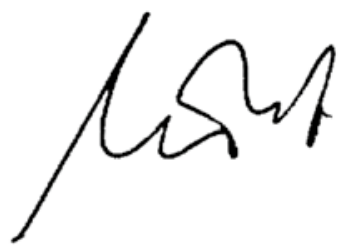

H. Siebert

\section{Korrespondenzadressen}

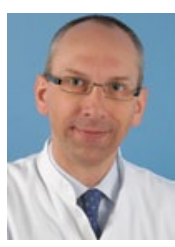

Prof. Dr. P.C. Strohm

Department Chirurgie,

Klinik für Orthopädie und

Unfallchirurgie,

Albert-Ludwigs-Universität

Freiburg

Hugstetter Straße 55,

79106 Freiburg

peter.strohm@uniklinik-

freiburg.de

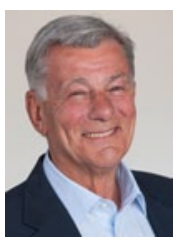

\section{Prof. Dr. H. Siebert}

Geschäftsstelle, Deutsche Gesellschaft für Orthopädie und Unfallchirurgie e.V. Berlin Straße des 17. Juni 106-108, 10623 Berlin

hsiebert@office-sha.de

\section{Einhaltung der ethischen Richtlinien}

Interessenkonflikt. P.C. Strohm und H. Siebert geben an, dass kein Interessenkonflikt besteht.

\section{FlinTIC-Studie auf dem Zivilschutzhubschrauber "Christoph 3" gestartet}

Traumapatienten versterben in der Frühphase in erster Linie aufgrund von Schädel-HirnTraumen und unkontrollierten Blutungen. Jüngere Untersuchungen, nicht zuletzt anhand von Datensätzen aus dem TraumaRegister $^{\circledR}$; der deutschen Gesellschaft für Unfallchirurgie, haben gezeigt, dass jeder dritte bis vierte Traumapatient bereits zum Zeitpunkt der Schockraumaufnahme eine signifikante Störung seiner Gerinnungsfunktion aufweist. Umgekehrt ist bekannt, dass eine frühe und aggressive Therapie der inzwischen als eigene Entität verstandenen akuten posttraumatischen Gerinnungsstörung das Überleben und Outcome von Traumapatienten wesentlich verbessern kann. Eine besondere Rolle spielt dabei die frühe und gezielte Substitution von Gerinnungsfaktoren.

\section{Die Pilotstudie}

Aktuell wird im Rahmen einer multizentrischen, doppelblinden und placebokontrollierten Pilotstudie geprüft, ob eine bereits im Rahmen der Prähospitalphase durchgeführte blutungshemmende Behandlung mit einem humanen Fibrinogenkonzentrat die Gerinnung bei Traumapatienten mit schwerer Blutung und/oder großem Blutverlust stabilisiert und Blutung und Blutverlust durch die ultrafrühe Gabe reduziert werden können (FlinTIC-Studie $=$ Faktor 1/Fibrinogen in Trauma Induced Coagulopathy). Außerdem wird sowohl die Sicherheit des Fibrinogens auf eine etwaige Erhöhung von Thrombosen hin untersucht, als auch die Frage ob der spätere Bedarf an Transfusionen und weiteren Blutprodukten (z. B. Blutplasma) gesenkt werden kann.

Eingeschlossen werden Traumapatienten mit schweren Blutungen oder Verdacht auf eine schwere innere Blutung. Die Patienten werden zu Vergleichszwecken entweder mit Fibrinogenkonzentrat oder Placebo behandelt. Bei Eintreffen im Krankenhaus wird die jeweilige Standardtherapie durchgeführt. Für diese Pilotstudie sind insgesamt 60 Patienten geplant. Die primäre Zielgröße der Studie ist die Veränderung der Kapazität der Plasma-Koagulation, gemessen anhand der Fibrinpolymerisation zum Zeitpunkt der Schockraumaufnahme.

\section{Weitere Stützpunkte gesucht}

Die Studie wird geleitet von Univ.-Prof. Dr. Dietmar Fries (Innsbruck); der nationale Koordinator für Deutschland ist Prof. Dr. Marc Maegele (Köln). Bislang werden Patienten an acht Rettungshubschrauberstützpunkten in Österreich eingeschlossen. Jüngst wurde der erste Stützpunkt in Deutschland am Luftrettungszentrum Köln mit dem Zivilschutzhubschrauber "Christoph 3" als Prüfstelle initiiert. Aktuell geplant ist der Studienstart an weiteren Stützpunkten in Deutschland und Tschechien. Bei Interesse bitte Kontaktaufnahme über den nationalen Koordinator.

\section{Kontakt: Prof. Maegele, maegelem@kliniken-koeln.de}

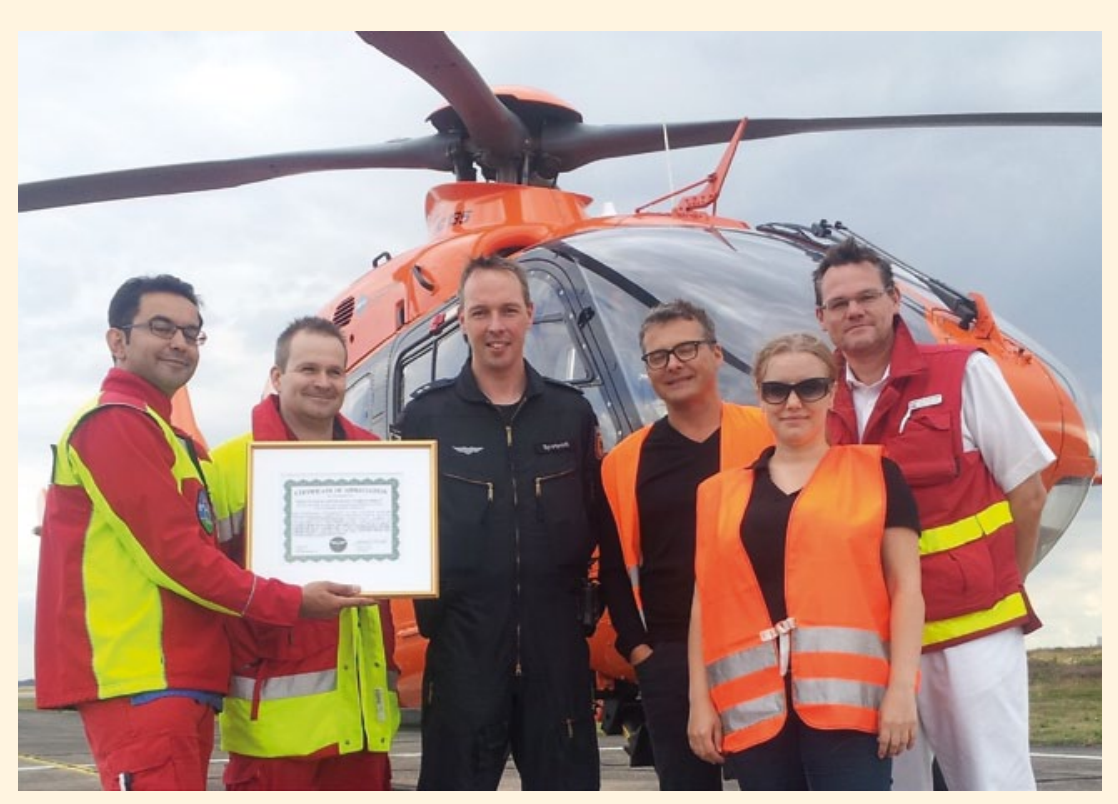

